\title{
The Effects of a Lifestyle Modification Program on a Leisure Physical Activity and Sedentary Behavior in a Brazilian Low Socioeconomic Community
}

\author{
Mariana Santoro Nakagaki ${ }^{1,2}$, Edilaine Michelin ${ }^{2}$ and Roberto Carlos Burini ${ }^{2}$ \\ 1. Center of Physical Education and Sport, Londrina State University (UEL), Londrina, Paraná, Brazil \\ 2. Center for Nutritional and Exercise Metabolism, Department of Public Health, School of Medicine (FMB), São Paulo State
} University (UNESP), Botucatu, São Paulo, Brazil

\begin{abstract}
Regular physical activity benefits the health of the general population, more in those with sedentary behaviors. PURPOSE: To verify the impact of adding leisure-time activities to the existed sedentary behaviors. METHODS: A cross-sectional and a longitudinal analysis were undertaken in an ongoing epidemiological study ("Move for Health") conducted by this Institution. The longitudinal study comprised 1572 subjects older than 35 yrs (53.8 $\pm 11.1 \mathrm{yrs}, 76 \%$ women) enrolled during the period of 2004 to 2015 . After a baseline assessment, the participants were submitted to a 10-week program of supervised physical exercises training and dietary counseling. The physical exercise protocol was composed by daily sessions (100 min), 3-5x/wk, including warm up/stretching (20min), 30 min walking (60\%-80\% $\left.\mathrm{VO}_{2 \mathrm{max}}\right)$, 40min strength in academy (3x 8-12 rep, 60\%-70\% 1RM) and stretching and cool down (10 min). By using the International Physical Activity Questionnaire (IPAQ-way long-version 8), it was evaluated the time spent on sedentary activities during the week and on weekends (h/day), in the domains of sedentary transport (h/week) and physical leisure activities (h/week) at baseline (M0) and after 10 weeks (M1) of physical intervention. The Student's t test was used to compare moments with a significance level of 5\%. RESULTS: I was shown that $18.3 \%$ presented lower values than $150 \mathrm{~min} / \mathrm{wk}$ of physical activity. Lower quartile of leisure domain on weekdays along with more sedentary behavior on weekends were determined by socioeconomic characteristics such as lower income and schooling. The sample referred low income ( $71 \%$ earning less than 5 minimum salary wage), low schooling (52.6\% uncompleted elementary school) but in a good-excellent status of health, according their self-perception. Leisure time-physical activity increased $2.12(P<0.001)$. Behaviors of sedentary transport and sitting time either weekdays or weekends did not change significantly $(P>0.05)$. CONCLUSION: An inexpensive and institutional-conducted lifestyle modification program like the "Move for Health" can provide extra energy expenditure, as leisure time, to a low socioeconomic community. However, it has been unsuccessfully proved to change other existing sedentary behavior.
\end{abstract}

Key words: Sedentary behavior, leisure time, physical activity.

\section{Introduction}

The physical inactivity is the fourth principal risk factor for global morbidity and mortality by noncommunicable diseases (NCD) [1]. According to IBGE [2], the proportion of adults classified as insufficiently active in Brazil was $46 \%$, more than half $(62.7 \%)$ of people aged over 60 was inactive and, the less sedentary group was 18 to 24 years old (36.7\%).

Corresponding author: Mariana Santoro Nakagaki, Ph.D. candidate, research field: lifestyle modification, sedentary behavior and metabolic syndrome.
Moreover, 50.6\% of individuals with no education or with uncompleted elementary grade were physically inactive.

One in two Brazilian and one in three worldwide population are considered physically inactive having $20 \%$ to $30 \%$ more chance to death in comparison with whom practice at least $30 \mathrm{~min} /$ day of moderate physical activity (PA) [1, 3, 4]. Hence, individuals moderately active when compared to completely inactive can reduce by $16 \%-30 \%$ all-cause mortality [5]. Overall, physical inactivity is acknowledged as the biggest public health problem of the 21st century [6]. 
Sedentary behavior is increasingly recognized as an important health determinant $[7,8]$ and currently, it has been also associated with increased risks of all-cause mortality [7]. Sedentary Behavior is described as any waking behavior characterized by an energy expenditure $\leq 1.5$ METs while in a sitting or reclining posture [9, 10]. Moreover, it is associated with environmental, socioeconomic and demographic factors [11]. Approximately 42.3 million people, or $28.9 \%$ of the adult population reported having seen television for 3 or more hours. As for the education level, the lower proportion of adults who watch television for three or more hours per day had a college degree, represented by $21.1 \%$ of this stratum [2].

The positive health effects of reducing a potentially damaging behavior, like sedentary time, might be dependent on the behavior with which it is replaced [12, 13]. There is a growing body of evidence suggesting that individuals of all ages should minimize their sedentary time (sitting) [14] and reduce sedentary behavior [15-17]. The beneficial effects of reducing sedentary behavior might be largely recognized to the resultant increase in time spent being active [18]. In past decades, with the advancement of technology it has been a declining in physical demands at work and for activities of daily living [19, 20], and the exercise during leisure time has been highlighted as an essential part of a healthy lifestyle by public health organizations [21, 22].

This study aims to verify the impact of adding leisure-physical activity protocol on the existing sedentary behaviors.

\section{Methods}

The program called "Move for Health" is an ongoing epidemiology study conducted, since 1991, by professionals linked to the Metabolism Exercise and Nutrition Center (CeMENutri) at UNESP Medical School. As a community based project it includes adult (> 35 yrs. old) participants from both genders that come to the clinic for preventive health examination and further interventions including nutrition reeducation, supervised physical exercise and other lifestyle factors.

They come to the clinic spontaneously or by friend or doctor indication looking for preventive health examination with further non-medicated interventions. Clinical, nutritional and fitness examinations, as well as physical exercises and dietary interventions are conducted by graduate students holding institutional fellowships linked to CeMENutri. The whole protocol lasts 10 weeks and is free of charge.

The first set of data of the present work came from baseline values of 1504 individuals enrolled to the program during 2007 to 2013. Evaluations were performed at the first contact with the participants applying a IPAQ (International Physical Activity Questionnaires) with questions about daily physical activity in four different domains: (work, leisure, transportation and domestic) beyond sedentary behavior. They were distributed according to their weekly physical activity in 3 groups (P25, P25-75 and P75) for both total and for its domains and analyzed with the co-variables gender, age, income, schooling and self-health perception.

The second set of data comprised 1572 subjects $(53.8 \pm 11.1 \mathrm{yrs}, 76 \%$ women) enrolled in the Program during the period of 2004 to 2015. After the baseline assessment the participants were submitted to a 10-week protocol of supervised physical exercises and dietary counseling (LSM). The physical exercise protocol was composed by daily sessions of $100 \mathrm{~min}$, 3-5x/wk, including $20 \mathrm{~min}$ warm up/stretching, $30 \mathrm{~min}$ walking $\left(60 \%-80 \% \quad \mathrm{VO}_{2 \max }\right), 40$ min strength in academy (3x 8-12 rep, 60\%-70\% 1RM) and 10min stretching and cool down. By using the International Physical Activity Questionnaire (IPAQ-way long-version 8), it was evaluated the time spent on sedentary activities during the week and on weekends (h/day), in the fields sedentary transport (h/week) and physical leisure activities (h/week) at baseline (M0) and after 10 weeks (M1) of physical intervention. The 
Student's t test was used to compare the two moments with a significance level of $5 \%$.

\section{Results}

The cross-sectional analysis of the baseline data showed $18.3 \%$ of the sample referring physical activity lower than $150 \mathrm{~min} / \mathrm{wk}$. The low physical activity levels were found on those with high schooling and high income. However, specifically in the leisure domain, the lower quartile was associated with lower income (Fig. 1) and schooling (Fig. 2). The lower leisure- physical activity were found either weekdays and weekends. On weekends it was associated with a more sedentary behavior (Table 1).

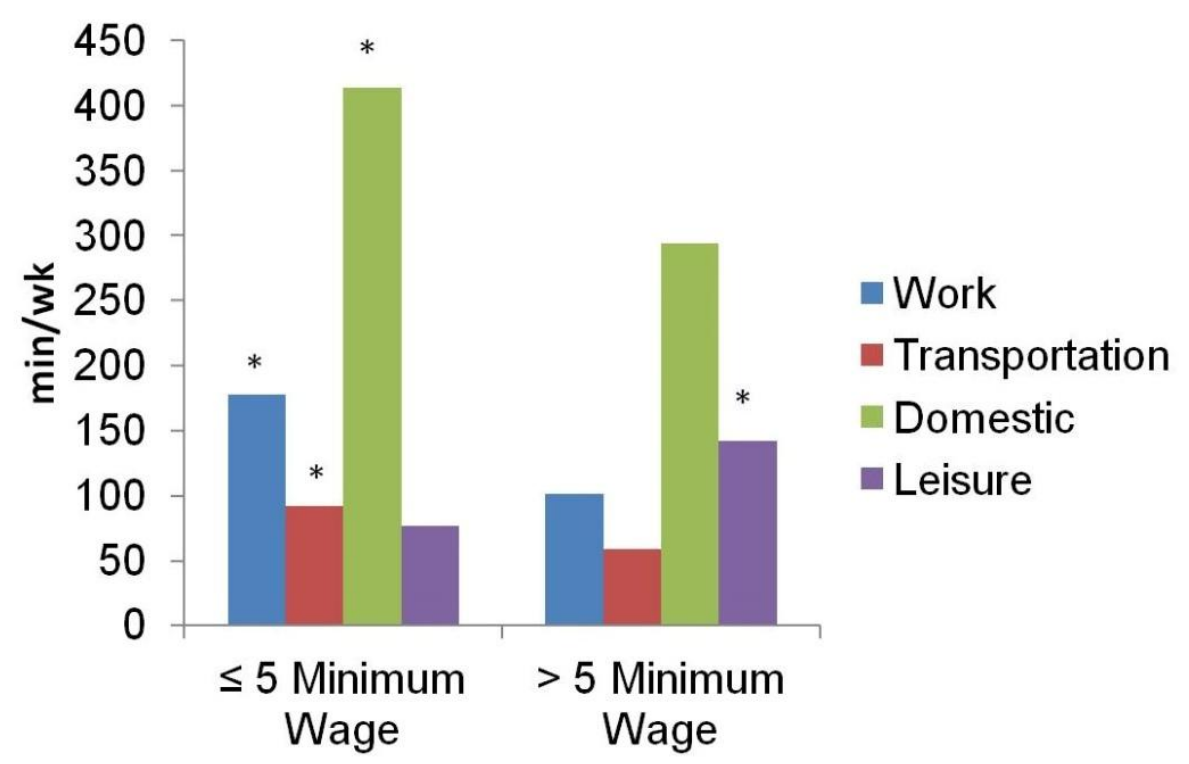

Fig. 1 Physical activity levels in different domains associated with income. *statistical significance $(P<0.05)$.

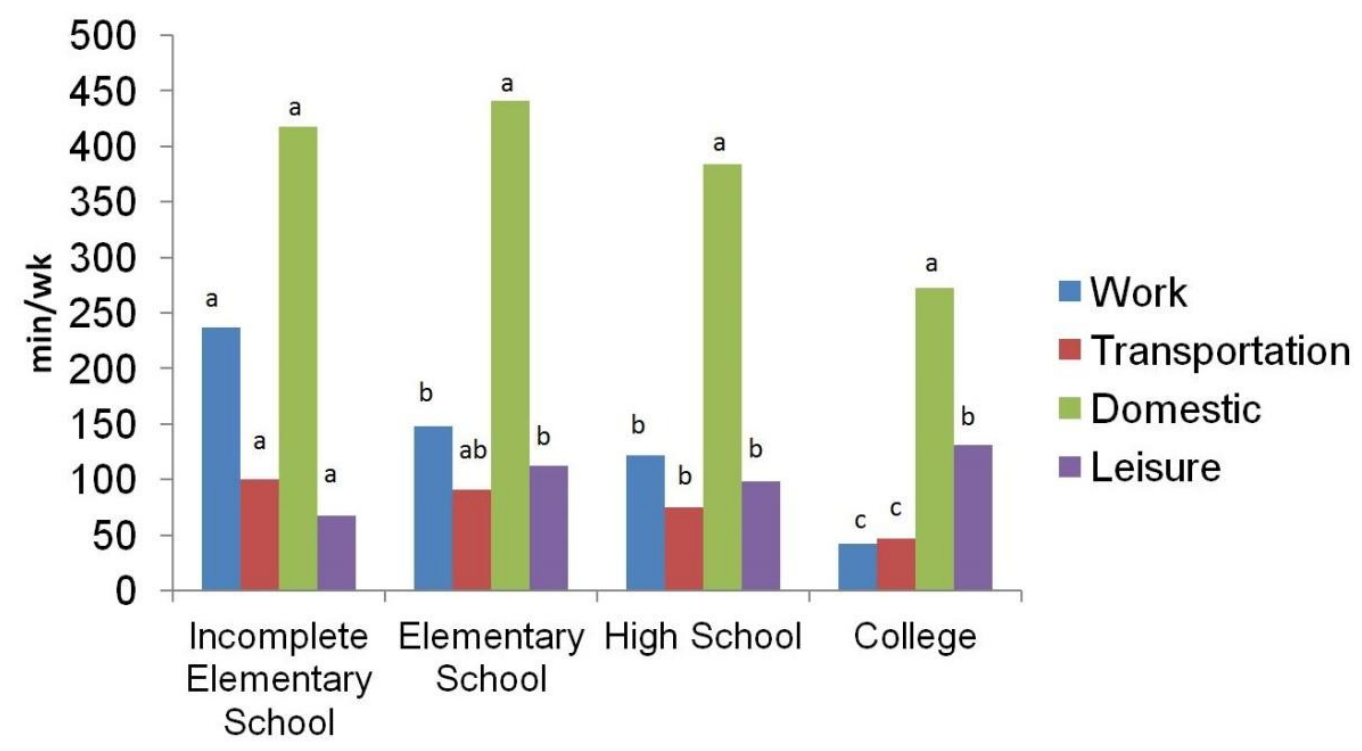

Fig. 2 Physical activity levels in different domains associated with schooling. Different letters show statistical significance $(P<0.05)$. 
Table 1 Sedentary behavior during week and during weekend associated with income and schooling.

\begin{tabular}{lll}
\hline & \multicolumn{1}{c}{ Sedentary behavior } \\
\cline { 2 - 3 } & During week (h/day) & \multicolumn{1}{c}{ During weekend (h/day) } \\
\cline { 2 - 3 } & Mean \pm SD $(\mathrm{N})$ & Mean \pm SD $(\mathrm{N})$ \\
\hline Income & $5.16 \pm 2.71(1022)$ & $5.65 \pm 3.20(1022)$ \\
\hline$\leq 5$ Minimum wage & $5.93 \pm 3.01(415)^{*}$ & $5.57 \pm 2.77(415)$ \\
\hline S Minimum wage & & $5.48 \pm 2.75(606) \mathrm{b}$ \\
\hline Schooling & $4.93 \pm 2.57(606) \mathrm{a}$ & $4.98 \pm 2.53(171) \mathrm{b}$ \\
Elementary school & $4.59 \pm 2.55(171) \mathrm{a}$ & $5.91 \pm 3.88(349) \mathrm{b}$ \\
High school & $5.58 \pm 2.80(349) \mathrm{b}$ & $5.89 \pm 2.90(332) \mathrm{b}$ \\
College & $6.45 \pm 3.11(332) \mathrm{c}$ & \\
\hline
\end{tabular}

*statistical significance $(P<0.05)$; different letters show statistical significance $(P<0.05)$.

After 10 weeks of intervention with the LSM there was a 2.12 fold increasing of physical activity (from $7.3 \mathrm{~h} / \mathrm{wk}$ to15.5h/wk $)(P<0.001)$. However, the individuals kept unchangeable their sedentary behaviors such as motor-vehicle transport $(4.55 \times$ $4.22 \mathrm{~h} / \mathrm{wk})$ and sitting time either on weekdays $(5.6 \times$ $5.5 \mathrm{~h} / \mathrm{d})$ or weekends $(5.6 \times 5.7 \mathrm{~h} / \mathrm{d})$.

\section{Discussion}

In Brazil, only $27.1 \%$ men aged 18 years or more practice the recommended level of physical activity during leisure time, while for women the percentage was even $18.4 \%$. The national average was $22.5 \%$ including urban and rural areas of the country. The percentage of adults who practiced the recommended level of physical activity during leisure time tended to decline with increasing age and increases with the level of education [2].

In the present case, the socioeconomic status determined the leisure pattern of physical activity. Less educated and lower income subjects had low leisure physical activity during working days, differently from higher educated-higher income subjects. This opposite behavior occurred also on weekends, with the former looking for more sedentary behaviors.

In the whole country, $14 \%$ of people 18 years or older were physically active at work. Among adults living in urban areas, $12.9 \%$ practiced 150 minutes of activity at work and among those living in rural areas were $21.1 \%$. The rate of men to this area was $22 \%$, while women was 7\%. This indicator was more representative of the people without education/ uncompleted elementary school and elementary school/ high school, with percentages of $17.3 \%$ and $17.4 \%$ in that order. From there, as the increased level of education, the percentages relating to this population showed declining [2].

The found socioeconomic pattern of lower physical activity behavior was expected. Lower schoolarity leads to less paid jobs and consequently a lower income. Additionally, less paid job usually requires high energy expenditure during the working hours added to the physical efforts of walking or biking as transportation and other non-automatic housework. Among white Brazilian adults, $28.7 \%$ practiced 30 minutes of physical activity in transportation. Among the blacks, this frequency was $38.3 \%$ and $33.9 \%$ among brown [2].

By spending high energy working hours during weekdays, it is expected that they look for light activities on weekends, reflecting a sedentary behavior. On the other hand, individuals with higher education end up getting higher paying jobs, bringing higher income. These individuals have automatic households and also their own vehicles as the main means of transport, leading to a sedentary behavior. In the domestic activities, it was estimated that $12.1 \%$ of people 18 years or older engaged in physical activity for at least 150 minutes a week, such as heavy cleaning or activities that require intense physical exertion. This indicator was strongly focused on the female audience 
in which $18.2 \%$ had 150 minutes of physical activity on household tasks, while the male audience, the practice of this activity to the recommended level was 5.4\% [2].

Some interrelated factors like aging, advancements of technologies, and changes in diets and lifestyles lead to a shift in the disease burden from infectious to noncommunicable diseases (NCDs) [23, 24].

The present data showed that our lifestyle modification program "Move for Health" would provide significant addition of leisure time of physical activity but was unable, in ten weeks, to modify the existing sedentary behavior of the participants. Thus, an inexpensive and institutional-conducted lifestyle modification program like the "Move for Health" can provide extra energy expenditure, as leisure time, to a low socioeconomic community. However, it has been unsuccessfully proved in changing other existing sedentary behavior.

\section{Reference}

[1] World Health Organization (WHO). 2009. "Global Health Risks: Mortality and Burden of Disease Attributable to Selected Major Risks." WHO Library Cataloguing-in-Publication Data. Accessed April 1, 2016. http://www.who.int/healthinfo/global_burden_disease/Gl obalHealthRisks_report_full.pdf

[2] Brazilian Institute of Geography and Statistics (IBGE). 2013. Research Board, Work and Income Coordination, National Health Survey. (in Portuguese)

[3] World Health Organization (WHO). 2011. "Noncommunicable Diseases Country Profiles." WHO Library Cataloguing-in-Publication Data, 207. Accessed October 19, 2015. http://www.who.int/nmh/publications/ncd_profiles_report .pdf

[4] Milanovic, Z., Pantelic, S., Trajkovic, N., Sporis, G., Kostic, R., and James, N. 2013. "Age-Related Decrease in Physical Activity and Functional Fitness among Elderly Men and Women." Clinical Interventions in Aging 8: 549-56.

[5] Pate, R. R., O’Neill, J. R., and Lobelo, F. 2008. "The Evolving Definition of Sedentary." Exerc. Sport Sci. Rev. 36 (4): 173-8.

[6] Blair, S. N. 2009. "Physical Inactivity: The Biggest Public Health Problem of the 21 st Century." Br. J. Sports Med. 43 (1): 1-2.

[7] Sedentary Behavior Research Network. 2012. "Letter to the Editor: Standardized Use of the Terms 'Sedentary' and 'Sedentary Behaviors'.” Appl. Physiol. Nutr. Metab. 37 (3): 540-2.

[8] Department of Health, Physical Activity, Health Improvement and Protection. 2011. Start Active, Stay Active: A Report on Physical Activity from the Four Home Countries' Chief Medical Officers. 33-4. Accessed December 9, 2015. https://www.gov.uk/government/uploads/system/uploads/ attachment_data/file/216370/dh_128210.pdf

[9] Biswas, A., Oh, P. I., Faulkner, G. E., Bajaj, R. R., Silver, M. A., Mitchell, M. S., and Alter, D. A. 2015. "Sedentary Time and Its Association with Risk for Disease Incidence, Mortality, and Hospitalization in Adults: A Systematic Review and Meta-Analysis." Ann. Intern. Med. 162 (2): 123-32.

[10] Thorp, A. A., Owen, N., Neuhaus, M., and Dunstan, D. W. 2011. "Sedentary Behaviors and Subsequent Health Outcomes in Adults a Systematic Review of Longitudinal Studies, 1996-2011." Am. J. Prev. Med. 41 (2): 207-15.

[11] Michelin, E., Corrente, J. E., and Burini, R. C. 2010. "Association of Physical Activity Levels with Socioeconomic, Obesity and Physical Fitness Indicators in Adults." Brazilian Journal of Physical Activity and Health 15 (1): 7. (in Portuguese)

[12] Owen, N., Salmon, J., Koohsari, M. J., Turrell, G., and Giles-Corti, B. 2014. "Sedentary Behaviour and Health: Mapping Environmental and Social Contexts to Underpin Chronic Disease Prevention.” Br. J. Sports Med. 48 (3): 174-7.

[13] Hamer, M., Stamatakis, E., and Steptoe, A. 2014. "Effects of Substituting Sedentary Time with Physical Activity on Metabolic Risk." Med. Sci. Sports Exerc. 46 (10): 1946-50.

[14] Chau, J. Y., der Ploeg, H. P., van Uffelen, J. G., Wong, J., Riphagen, I., Healy, G. N., Gilson, N. D., Dunstan, D. W., Bauman, A. E., Owen, N., and Brown, W. J. 2010. “Are Workplace Interventions to Reduce Sitting Effective? A Systematic Review." Prev. Med. 51 (5): 352-6.

[15] Evans, R. E., Fawole, H. O., Sheriff, S. A., Dall, P. M., Grant, P. M., and Ryan, C. G. 2012. "Point-of-Choice Prompts to Reduce Sitting Time at Work: A Randomized Trial." Am. J. Prev. Med. 43 (3): 293-7.

[16] Stephens, S. K., Winkler, E. A., Trost, S. G., Dunstan, D. W., Eakin, E. G., Chastin, S. F. M., and Healy, G. N. 2014. "Intervening to Reduce Workplace Sitting Time: How and When Do Changes to Sitting Time Occur?" Br. J. Sports Med. 48 (13): 1037-42.

[17] Gomersall, S. R., Norton, K., Maher, C., English, C., and Olds, T. S. 2014. "In Search of Lost Time: When People Undertake a New Exercise Program, Where Does the 


\section{Sedentary Behavior in a Brazilian Low Socioeconomic Community}

Time Come From? A Randomized Controlled Trial." J. Sci. Med. Sport 18 (1): 43-8.

[18] Saunders, T. J., Larouche, R., Colley, R. C., and Tremblay, M. S. 2012. "Acute Sedentary Behaviour and Markers of Cardiometabolic Risk: A Systematic Review of Intervention Studies." J. Nutr. Metab. 2012: 1-12.

[19] Archer, E., Shook, R. P., Thomas, D. M., Church, T. S., Katzmarzyk, P. T., Hebert, J. R., Mclver, K. L., Hand, G. A., Lavie, C. J., and Blair, S. N. 2013. “45-Year Trends in Women's Use of Time and Household Management Energy Expenditure." Plos One 8 (2): e56620.

[20] Church, T. S., Thomas, D. M., Tudor-Locke, C., Katzmarzyk, P. T., Earnest, C. P., Rodarte, R. Q., Martin, C. K., Blair, S. N., and Bouchard, C. 2011. "Trends over 5 Decades in U.S. Occupation-Related Physical Activity and Their Associations with Obesity." Plos One 6 (5): e19657.

[21] Haskell, W. L., Lee, I. M., Pate, R. R., Powell, K. E., Blair, S. N., Franklin, B. A., Macera, C. A., Heath, G. W.,
Thompson, P. D., and Bauman, A. 2007. "Physical Activity and Public Health: Updated Recommendation for Adults from the American College of Sports Medicine and the American Heart Association." Med. Sci. Sports Exerc. 39 (8): 1423-34.

[22] World Health Organization (WHO). 2010. Global Recommendations on Physical Activity for Health. Geneva (Switzerland): WHO Press, 24-6. Accessed October 19, 2015.

http://apps.who.int/iris/bitstream/10665/44399/1/9789241 599979_eng.pdf

[23] Murray, S. 2006. "Doubling the Burden: Chronic Disease." Can. Med. Assoc. J. 174 (6): 771.

[24] Yusuf, S., Reddy, S., Ounpuu, S., and Anand, S. 2001. "Global Burden of Cardiovascular Diseases: Part I: General Considerations, the Epidemiologic Transition, Risk Factors, and Impact of Urbanization." Circulation 104 (22): 2746-53. 